\title{
Using Page's Cumulative Sum Test on MODIS time series to detect land cover changes
}

\author{
T.L. Grobler, Student Member, IEEE, E.R. Ackermann, Student Member, IEEE, A.J. van Zyl, J.C. Olivier, \\ W. Kleynhans and B.P. Salmon
}

\begin{abstract}
Human settlement expansion is one of the most pervasive forms of land cover change in South Africa. The use of Page's Cumulative Sum Test is proposed as a method to detect new settlement developments in areas that were previously covered by natural vegetation using $500 \mathrm{~m}$ MODIS time series satellite data. The method is a sequential per pixel change alarm algorithm that can take into account positive detection delay, probability of detection and false alarm probability to construct a threshold. Simulated change data was generated to determine a threshold during a preliminary off-line optimization phase. After optimization the method was evaluated on examples of known land cover change in the Gauteng and Limpopo provinces of South Africa. The experimental results indicated that CUSUM performs better than band differencing in the before mentioned study areas.
\end{abstract}

\section{INTRODUCTION}

Remotely sensed satellite data provide researchers with an efficient way to monitor and detect land cover changes in a way that has not been possible in the past [1]. One way of accomplishing this is to compare high resolution images acquired at different times. Based on a change metric and threshold selection method, each pixel is classified as either being a change or no-change pixel. However, using only two images can lead to suboptimal results, as similar land cover types can vary significantly during various stages of the natural growth seasonal cycle [2]. To address this problem it was proposed that the sample rate of medium resolution remote sensing data acquisitions should be high enough to ascertain change events from natural phenological cycles [3], [4]. The Moderate-resolution Imaging Spectroradiometer (MODIS) data product MCD43A4 (used in this study) uses daily Terra and Aqua satellite overpasses to produce a 500 meter resolution conglomerated image every 8 days, and as such offers a high temporal frequency, which makes it possible to effectively detect changes by using time-series analysis.

T.L. Grobler is with the Department of Electrical, Electronic and Computer Engineering, University of Pretoria, Pretoria 0002, South Africa and also with Defence, Peace, Safety and Security, Council for Scientific and Industrial Research, Pretoria 0001, e-mail: tgrobler@csir.co.za.

E.R. Ackermann is with the Computational and Applied Mathematics Department, Rice University, Houston, TX 77005-1827 USA

A.J. van Zyl is with the Department of Mathematics and Applied Mathematics, University of Pretoria, Pretoria 0002, South Africa.

J.C. Olivier is with the School of Engineering, University of Tasmania, 7001, Hobart, Australia.

W. Kleynhans and B.P. Salmon is with the Remote Sensing Research Unit, Meraka Institute, Council for Scientific and Industrial Research, Pretoria 0001, South Africa and with the Department of Electrical, Electronic and Computer Engineering, University of Pretoria, Pretoria 0002, South Africa

Manuscript received ???, 2011.
The traditional band differencing algorithm [2], the autocorrelation technique [5], the predictive modeling approach [6], [7], the recursive merging algorithm [6] and the FFT (Fast Fourier Transform) sliding window [8] are some of the high temporal time-series change detection algorithms that have been successfully used in a remote sensing context. Most of the remote sensing time-series change detection algorithms in the literature use some form of windowing, in other words only recent data is used to detect change [6]. In contrast the algorithm proposed in this paper is windowless, and as such there is no step required to determine the window length. The problem of using only recent data, which was extracted using a window, is that the average pixel behavior might not be captured if the window is not long enough. For example, a windowed algorithm will be more prone to classify a vegetation pixel experiencing a drought as a change pixel, when in fact the pixel did not change from vegetation to settlement.

In this paper we argue that there are several metrics by which a change detection algorithm should be evaluated. An obvious one is detection delay, by which we mean the time taken for the change detection algorithm to declare that a change has occurred, given that a change in the data actually occurred. Then there is the question of how likely it is for the algorithm to declare that a change has occurred given that the change in the data did in fact occur, a metric we refer to as either probability of detection or the true positive rate (TP). There are more metrics that need to be consider. For example, there is the possibility that the algorithm will declare change, given that change has not occurred in the data, which we can refer to as either the probability of false detection (alarm) or the false positive rate (FP). As this paper is presented in a statistical framework we prefer not to adopt the detection theory terms TP and FP. Then there is the question of how to eliminate the need for a windowing mechanism, in the sense that our algorithm is on-line or sequential, i.e. it uses all the past data. This is possible when the algorithm has the property that it only starts behaving differently when an actual change occurred. However, it is not common for the above four change detection criteria to be considered simultaneously in a remote sensing change detection context, and in that respect this paper will present novel results since the paper will show how to sequentially detect change (vegetation pixels that are changed into settlement pixels) as accurately and quickly as possible, while staying below a certain probability of false alarm.

The objective of this paper is to use the Cumulative Sum (CUSUM) as a change detection algorithm in Page's original 
form [9] in order to process samples sequentially. Windowed versions of the CUSUM algorithm have been used with MODIS in the past, typically in a bootstrapping [10] or in an in-control process mean context [6], [11]. We can implement CUSUM without using a window, because in Page's original form the CUSUM statistic is derived from log-likelihood ratios which can be obtained from densities estimated at every timestep of the year. The densities at each time-step thus circumvents intra-annual variation. The densities are constructed by using the Colored Simple Harmonic Oscillator (CSHO) which can replicate average pixel behavior which implies that the effect of inter-annual variation is also minimized [12].

\section{DATA DESCRIPTION}

The input time series data is extracted from the 8 day composite MODIS MCD43A4 Bidirectional Reflectance Distribution Function (BRDF) corrected $500 \mathrm{~m}$ land surface reflectance product corresponding to a total area of approximately $230 \mathrm{~km}^{2}$ in Gauteng and $800 \mathrm{~km}^{2}$ in Limpopo, South Africa ${ }^{1}$. The temporal acquisition rate of MODIS MCD43A4 roughly translates to 45 observations per year. The most pervasive form of land cover change in South Africa is that of settlement expansion. Two land cover classes are considered: settlements and natural vegetation, denoted by $s$ and $v$ respectively. In this study the settlements class contains pixels that contain more than $50 \%$ buildings, whereas the vegetation class contains pixels with more than $90 \%$ vegetation. The Gauteng data set consists of 1106 MODIS pixels, while the Limpopo data set contains 3349 MODIS pixels and was selected with visual (human) interpretation of two high resolution Système Probatoire d'Observation de la Terre (SPOT) images from the year 2001 and 2009. We selected MODIS pixels that, according to the SPOT images, either did not change or changed from vegetation to settlement. Each MODIS pixel contains eight time series (seven MODIS land bands, and NDVI) with $I=368$ observations (extracted between January 2001 and March 2009). The NDVI time series was computed using the first two spectral land bands. The Gauteng and Limpopo datasets are respectively divided into the three classes: natural vegetation (592 Gauteng pixels and 1497 Limpopo pixels), settlements (333 Gauteng pixels and 1735 Limpopo pixels) and real land cover change from vegetation to settlement (181 Gauteng pixels and 117 Limpopo pixels) [12].

\section{Page's Cumulative Sum Test}

Consider a measurable space $(\Omega, \mathcal{F})$, consisting of a sample space $\Omega$ and a $\sigma$-field $\mathcal{F}$ of events [13]. Further consider a family $\left\{P_{\tau} \mid \tau \in[1,2, \cdots, \infty]\right\}$ of probability measures on $(\Omega, \mathcal{F})$ and a random sequence $z=\left\{z_{k} ; k=1,2, \cdots \infty\right\}$, such that, under $P_{\tau}, z^{-\tau}=\left\{z_{1}, z_{2}, \cdots, z_{\tau-1}\right\}$ are independent and identically distributed (i.i.d.) with a fixed marginal distribution $Q_{0}$ and $z^{+\tau}=\left\{z_{\tau}, z_{\tau+1}, \cdots, \infty\right\}$ are i.i.d. with marginal distribution $Q_{1}$ and are independent of $z^{-\tau}$. The probability densities associated with $Q_{0}$ and $Q_{1}$ are $q_{0}$ and $q_{1}$ respectively. We would like to consider a procedure that

\footnotetext{
${ }^{1}$ The MODIS MCD43A4 product can be downloaded from http://modis.gsfc.nasa.gov/data/.
}

can detect a change in the underlying distribution of $z_{k}$ (when $z_{k}$ gets sampled from $Q_{1}$ instead of $Q_{0}$ ), if it occurs (i.e. if $\tau<\infty$ ), as quickly as possible after it occurs. As a set of detection strategies, it is natural to consider the set $\mathcal{T}$ of all (extended) stopping times with respect to the filtration $\left\{\mathcal{F}_{k}\right\}$, where $\mathcal{F}_{k}$ denotes the smallest $\sigma$-field with respect to which $z_{0}, z_{1}, \cdots, z_{k}$ are measurable. Thus, when the stopping time $T$ takes on the value $k$, the interpretation is that $T$ has detected the existence of a change point $\tau$ at or prior to time $k$. It is of interest to penalize expected delay via its worst case value

$$
d(T)=\sup _{\tau \geq 1} \operatorname{ess} \sup \mathbb{E}_{\tau}\left\{(T-\tau+1)^{+} \mid \mathcal{F}_{\tau-1}\right\},
$$

where $\mathbb{E}_{\tau}\{\cdot\}$ denotes expectation under the distribution $P_{\tau}$ and $(T-\tau+1)^{+}=\max \{T-\tau+1,0\}$. Note that ess sup $\mathbb{E}_{\tau}\{(T-$ $\left.\tau+1)^{+} \mid \mathcal{F}_{\tau-1}\right\}$ is the worst case average delay under $P_{\tau}$, where the worst case is taken over all realization of $z^{-\tau}$. The desire to make $d(T)$ small must be balanced with a constraint on the false alarm rate. We accept the fact that false alarms will occur, however we want to fix the rate at which they occur. The false alarm rate is quantified by the mean time between false alarms

$$
f(T)=\mathbb{E}_{\infty}\{T\} .
$$

A useful design criterion is then given by

$$
\inf _{T \in \mathcal{T}} d(T) \text { subject to } f(T) \geq \lambda,
$$

where $\lambda$ is a positive finite constant. A stopping time is desired that minimizes the worst case expected delay within a lowerbound constraint on the mean time between false alarms. An algorithm that meets the requirements of (3) is Page's CUSUM test [13]. In particular, for $h \geq 0$ the CUSUM stopping time is defined as

$$
T_{h}^{\text {CUSUM }}=\inf \left\{k \geq 0 \mid g_{k} \geq h\right\},
$$

where

$$
g_{k}= \begin{cases}\left(g_{k-1}+s_{k}\right)^{+} & \text {if } k>0 \\ y \in \mathbb{R}^{+} & \text {if } k=0\end{cases}
$$

and

$$
s_{k}=\ln \frac{q_{1}\left(z_{k}\right)}{q_{0}\left(z_{k}\right)} .
$$

Under normal CUSUM operating conditions $y$ is set to 0 .

\section{APPlying CUSUM to MODIS}

Assume that an observed MODIS pixel $\mathcal{Z}^{c_{0}}=$ $\left\{\mathbf{z}_{k}^{c_{0}}\right\}_{k=\{1,2, \cdots\}}$ belongs to class $c_{0} \in \mathcal{C}$. With $\mathbf{z}_{k}^{c_{0}}$ defined as $\left\{z_{k}^{c_{0}, b}\right\}_{b \in\{1 \cdots 7, \mathrm{NDVI}\}}$, where $b$ represents the MODIS band (seven land bands or NDVI). Each band can also be denoted separately with $\mathcal{Z}^{c_{0}, b}=\left\{z_{k}^{c_{0}, b}\right\}_{k=\{1,2, \cdots\}}$. It is assumed that at some point in time $\tau$ the observed MODIS pixel changes into another class $c_{1} \in \mathcal{C}$.

Under the assumption of spatial independence, each observed signal in MODIS band $b$ belonging to the same class $c \in \mathcal{C}$ is a sample path of a stochastic process $\left\{Z_{k}^{c, b}\right\}_{k=\{1,2, \cdots\}}$. Since $\left\{Z_{k}^{c, b}\right\}_{k=\{1,2, \cdots\}}$ is a stochastic process its first order statistical description can be determined. 
The first order statistical description (also known as the time varying model [14]) is equivalent to the set of probability density functions at each time step $k,\left\{q_{k}^{c, b}\right\}_{k=\{1,2 \cdots\}}$. If we assume that the MODIS data contain no inter annual variation, in other words we assume that MODIS time series is periodic (45 observations in a year), then we have that $q_{k}^{c, b}=q_{k+45}^{c, b}$. To estimate $q_{i}^{c, b}$ at time of the year $i$ we first group all the observations of a particular time of the year together with

$$
\mathcal{G}_{i}^{c, b}=\operatorname{pr}_{i+45 n} \mathcal{Z}^{c, b}, n=0,1, \cdots, N, 1 \leq i \leq 45,
$$

where $\mathrm{pr}$ is the projection operator and $N$ is the number of years. We can determine $\mathcal{G}_{i}^{c, b}$ for each observed pixel in class $c$. After unifying all the computed $\mathcal{G}_{i}^{c, b}$ we can estimate $q_{i}^{c, b}$ using kernel density estimation. We used a Gaussian kernel with Silverman's rule of thumb as bandwidth selection rule, since the densities appear to be almost Gaussian ${ }^{2}$.

We argue that for the classes used in this study we can ignore the effect of spatial correlation as the classes are homogeneous and are restricted to reasonably small study areas. The densities are constructed from either true or simulated data. As our ground truth data (input MODIS time series) is limited we employ simulated data to construct the densities. We employ a trained CSHO to generate independent sample paths of each class. The ground truth data contain high spatial correlation and spatial dependence, since the pixels are all taken from the same study area. The CSHO only mimics limited spatial correlation by replicating the parameters of each class (for instance the sample paths of the CSHO are reasonably in phase, have slight differences in long term mean and seasonal amplitude). Since the CSHO mimics correlation through the features of a class it provides a spread of mean and amplitude for a specific class. The CSHO is less correlated than the ground truth data, which removes some of the bias that would be contained in the densities created directly from the ground truth data. For non-homogeneous classes we would have to employ smaller neighborhoods in each class to obtain better approximations for the individual time-step densities.

The classes (data sets) under consideration are almost cyclostationary and as such an assumption of zero inter-annual variation is acceptable [12]. The CSHO also minimizes the error incurred due to inter-annual variation as it generates the average behavior of a class in an average year [12].

To be able to use CUSUM on each MODIS band we have to adapt (6) in the following way

$$
s_{k}^{b}=\ln \frac{q_{k}^{c_{1}, b}\left(z_{k}^{b}\right)}{q_{k}^{c_{0}, b}\left(z_{k}^{b}\right)} .
$$

Using (8) we can detect when an observed MODIS pixel in band $b$ changes from class $c_{0}$ to $c_{1}$ sequentially. The class superscript is dropped from the observation $z_{k}^{b}$ if we do not know whether a pixel changed or not.

Note that (8) violates the identically distributed assumption of CUSUM, since $q_{k}^{b} \neq q_{j}^{b} \forall(k-j) \bmod 45 \neq 0$. To apply (8) we also need to assume independent observations, which

\footnotetext{
${ }^{2}$ The densities were estimated via the KDE Matlab toolbox which can be obtained from http://www.ics.uci.edu/ ihler/code/kde.html.
}

is not the foremost assumption for MODIS [14]. Due to the shortcomings introduced by (8) and the fact that the densities are estimated, the optimality of our results can no longer be guaranteed by the CUSUM algorithm.

\section{Justification of Approach And Alternatives}

CUSUM can be implemented (in the remote sensing field) with or without a window. In cases where the data exhibit severe underlying trends a windowed CUSUM approach based on an in-control process mean model is a better alternative, since such an algorithm would be better suited to incorporate underlying trends [6], [11]. The one approach is not superior to the other approach, especially since both approaches require training data. Choosing a windowed or windowless approach depends completely on the application. The $2 \times 45$ non-parametric densities proved sufficient in implementing CUSUM sequentially, but it is not the only possibility. We specifically chose the $2 \times 45$ non-parametric saturated densities as our underlying model to ensure maximum flexibility. The densities were built up with a trained CSHO simulator. As the densities appear Gaussian for our case study a good simplification (alternative) would be to use $2 \times 45$ parametric Gaussian densities instead. As the means in our case study appear to be sinusoidal [12], a further simplification would be to parameterize the mean and variance using harmonic models to avoid using 45 different values for the mean and variance respectively. The log-likelihood deviation measure was used as an effective compact aggregate way of detecting mean (level) changes, while incorporating seasonal variation. Instead of using log-likelihood deviations, residuals or squared residuals (centered by the variance) could have been used to detect mean and variance changes respectively [11]. Another alternative change detection algorithm is to detect changes in the first few harmonic components of a MODIS time series [8].

\section{BAND DIFFERENCING}

The CUSUM method was compared to another threshold approach that utilizes the high temporal resolution time-series data provided by MODIS. This computationally parsimonious change detection method was proposed by Lunetta et al. [2] with threshold $z$ (we will use $\delta$ instead to avoid ambiguity).

\section{ExPERIMENTAL Results: GAUteng CASE Study}

In this section we apply CUSUM on MODIS data in order to detect when vegetation pixels in the study areas change into settlement pixels. The result section is divided into two subsections. In the first subsection we present an offline optimization algorithm to determine the best threshold $h$ by performing a sweep of $h$ from 1 to 100 on simulated data to establish an intuitive base of the performance of CUSUM on MODIS data in the study areas. The simulated data that we used were generated by the CSHO [12]. In the second subsection we analyze the performance of the off-line determined $h$ on real world MODIS change data and compare it to the band differencing method (on the same data). 
A. The off-line training of CUSUM change detection using simulated MODIS data

We use $P_{\mathrm{D}}, P_{\mathrm{FA}}$ and $\mathbb{E}\left\{(T-\tau)^{+368}\right\}$ as metrics instead of $d(T)$ and $f(T)$, since we need to use metrics that can be fairly compared to non sequential change detection algorithms. Here $P_{\mathrm{D}}$ is the probability of correctly detecting a change within the 8 year observation period, $P_{\mathrm{FA}}$ is the probability of detecting a change when there was no change in the 8 year period and $\mathbb{E}\left\{(T-\tau)^{+368}\right\}=\mathbb{E}\{\min \{\max \{T-\tau, 0\}, 368-\tau\}\}$ is the positive expected delay truncated to 368 observations.

We propose the following algorithm, with input vector $(j, k, l, m, n)$, to determine the best threshold $h$ :

1) Use $j$ pixels of the no change vegetation data (real world no-change data) to learn the parameters needed by the simulator (training set).

2) Use $k$ no change settlement pixels (real world no-change data) to estimate the parameters needed by the simulator (training set).

3) Now using the trained simulator, simulate $l$ pixels of each class, and use them to create the 45 probability density functions that span a year.

4) Simulate $m$ pixels of each class, and use those to create simulated change data, where the change point $\tau$ has density $\mathrm{U}[1,300]$. The change is simulated by using linear blending over a 6 month period [5] ${ }^{3}$.

5) Simulate $n$ pixels of no change vegetation pixels.

6) For each threshold $h$ perform the CUSUM algorithm on each band and determine $P_{\mathrm{D}}, P_{\mathrm{FA}}$ and $\mathbb{E}\left\{(T-\tau)^{+368}\right\}$ using the simulated change and no change data.

7) To determine the best $h$ for each band calculate the $\kappa$ coefficient (based on the number of correctly detected changes and the number of incorrectly detected changes) at each $h$ in the sweeping interval and then select the $h$ value that produces the largest $\kappa$ coefficient.

There is no need to perform multiple experiments as [12] showed that the differences between simulated batches of pixels are statistically insignificant. The resulting $P_{\mathrm{D}}, P_{\mathrm{FA}}$ and $\mathbb{E}\left\{(T-\tau)^{+368}\right\}$ metrics, determined for the Gauteng data set with input vector $(592,333,2000,3000,3000)$, are shown in Figure 1.

Figures 1a to $1 \mathrm{c}$ indicates that the best possible CUSUM threshold $h$, is different for each band. The value of $h$ is dependent on two factors, the amount of dependence in the data and the separability of the two classes. The higher the dependence the higher the noise floor of $g_{k}^{b}$ (quantity (5) for band $b$ ), which forces $h$ to be large in order to keep the false alarm probability low. A large $h$ value increases the detection delay. The higher the separability the larger $h$ can be as the growth rate of $g_{k}^{b}$ will be steeper after the change point. Effectively we would like to choose the bands which are the most separable while also exhibiting low dependence. The bands $\{1,2,3,4, \mathrm{NDVI}\}$ are either highly separable or are less dependent on previous observations (some even have both properties) and as it turns out also perform better than the other MODIS bands (see Figure 1d). The

\footnotetext{
${ }^{3}$ The way that the blending is done is actually quite arbitrary and doesn't influence the results of this paper.
}

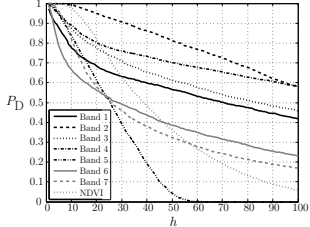

(a) $P_{\mathrm{D}}$ versus $h$

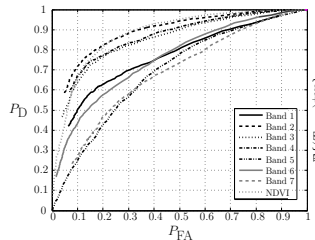

(c) $\mathbb{E}\left\{(T-\tau)^{+368}\right\}$ versus $h$.

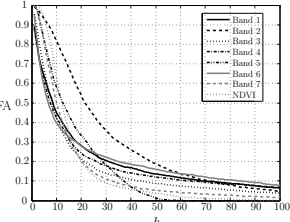

(b) $P_{\mathrm{FA}}$ versus $h$.

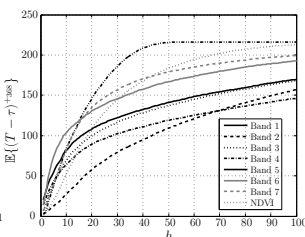

(d) $P_{\mathrm{D}}$ versus $P_{\mathrm{FA}}$.
Fig. 1: Measured $P_{\mathrm{D}}, P_{\mathrm{FA}}$ and $\mathbb{E}\left\{(T-\tau)^{+368}\right\}$ values for the simulated data in Gauteng.

dependence can be estimated via the $\lambda$ parameter of the CSHO, while the separability can be estimated by determining the Hellinger distance between the time varying models. It is worth mentioning that the Hellinger distance in each band is not constant and various during the year, which means that $g_{k}^{b}$ will grow at different rates during different times of the year. From a physical perspective it makes sense that bands $\{1,2,3,4$, NDVI $\}$ would perform well as vegetation display unique identifiable characteristics in the visible and near-infrared regions of the electromagnetic spectrum.

The most important graph in Figure 1 is the Receiver Operating Characteristics (ROC) curve (Figure 1d), since it displays the probability of correctly detecting a change in the 8 year observation period against declaring a change during the observation period if none occurred. Furthermore we can see from Figure 1c that the best threshold induces an upper positive expected delay of about 3 years before a change is detected. We also see that the detection performance for a 2 year delay is sufficient, however a 1 year delay causes too many false alarms.

\section{B. The performance of CUSUM on real world MODIS data}

To evaluate the performance of CUSUM on the real world data we used the metrics $P_{\mathrm{D}}$ and $P_{\mathrm{FA}}$. We could not measure any form of delay, as the true change point for the real world change data was unknown.

To summarize, we propose the following methodology to determine the effectiveness of CUSUM on the real world change data set:

1) Use the off-line optimization algorithm of the previous section with input vectors $(296,333,1000,1000,1000)$ and $(749,1735,1000,1000,1000)$ for the Gauteng and Limpopo data sets respectively, to determine the threshold $h$ for each study region. Note that only $50 \%$ (random $50 \%$ ) of the no change vegetation data was used to learn the parameters needed by the simulator and $50 \%$ of the real data was left for validation. 
2) Apply the best $h$ value on the no change real vegetation data (validation data set) and the real change data to determine $P_{\mathrm{D}}$ and $P_{\mathrm{FA}}$ (for each study region).

Cross validation was performed by repeating the above experiment multiple times. We also repeated the above experiment under a 5\% mislabeling assumption. We also used a training dataset and a validation data set (equal in size) which were the least correlated with each other (from all possible training and validation data sets). Both additional scenarios produced statistically insignificant deviations from the standard crossvalidation experiment, indicating that the algorithm is robust against mislabeling and that the classes are in fact homogenous. The results of the standard cross-validation procedure (50 experiments) is shown in Table I.

TABLE I: CUSUM applied on MODIS data

\begin{tabular}{|c|c|c|c|c|c|c|}
\hline $\begin{array}{l}\text { MODIS band } \\
\frac{\text { Gauteng }}{\text { Limpopo }}\end{array}$ & $h$ & $\sigma_{h}$ & $P_{\mathrm{D}}$ & $\sigma_{P_{\mathrm{D}}}$ & $P_{\text {FA }}$ & $\sigma_{P_{\mathrm{FA}}}$ \\
\hline \multirow{2}{*}{1} & 38.18 & 6.65 & 0.9829 & 0.0051 & 0.1896 & 0.0430 \\
\hline & 49.14 & 9.53 & 0.7720 & 0.0215 & 0.2536 & 0.0320 \\
\hline \multirow{2}{*}{2} & 53.73 & 5.01 & 0.8731 & 0.0039 & 0.1304 & 0.0234 \\
\hline & $\overline{40.61}$ & $\overline{7.54}$ & $\overline{0.6641}$ & 0.0210 & $\overline{0.3008}$ & 0.0340 \\
\hline \multirow{2}{*}{3} & 28.39 & 4.61 & 0.9846 & 0.0023 & 0.1748 & 0.0336 \\
\hline & $\overline{34.06}$ & $\overline{6.43}$ & $\overline{0.6764}$ & $\overline{0.0321}$ & $\overline{0.2039}$ & $\overline{0.0267}$ \\
\hline \multirow{2}{*}{4} & 39.13 & 7.56 & 0.9835 & 0.0008 & 0.1559 & 0.0253 \\
\hline & 36.59 & 9.27 & 0.6932 & 0.0241 & 0.2028 & 0.0267 \\
\hline \multirow{2}{*}{5} & 16.53 & 3.04 & 0.7874 & 0.0646 & 0.5406 & 0.0562 \\
\hline & $\overline{23.56}$ & $\overline{5.01}$ & $\overline{0.6713}$ & $\overline{0.0252}$ & $\overline{0.4383}$ & $\overline{0.0352}$ \\
\hline \multirow{2}{*}{6} & 17.17 & 4.44 & 0.2234 & 0.0783 & 0.3561 & 0.0718 \\
\hline & $\overline{17.49}$ & 6.33 & $\overline{0.7079}$ & 0.0416 & $\overline{0.4821}$ & 0.0485 \\
\hline \multirow{2}{*}{7} & 19.42 & 3.38 & 0.7178 & 0.0632 & 0.2508 & 0.0892 \\
\hline & $\overline{39.10}$ & $\overline{7.73}$ & $\overline{0.7239}$ & 0.0307 & $\overline{0.3210}$ & 0.0456 \\
\hline \multirow{2}{*}{ NDVI } & 20.91 & 1.88 & 0.8354 & 0.0478 & 0.1724 & 0.0806 \\
\hline & 35.35 & $\overline{5.00}$ & $\overline{0.7544}$ & $\overline{0.0471}$ & $\overline{0.4390}$ & $\overline{0.0501}$ \\
\hline
\end{tabular}

Again we see that $\{1,2,3,4$, NDVI $\}$ give the best results. The Gauteng dataset (band 4 was the best band) performs better than the Limpopo dataset (band 1 performed the best), since vegetation and settlement is more separable in Gauteng (a lot of residual vegetation is found in the settlement class of Limpopo) [12].

TABLE II: Band differencing applied on MODIS data

\begin{tabular}{|c|c|c|c|}
\hline $\begin{array}{l}\text { MODIS band } \\
\frac{\text { Gauteng }}{\text { Limpopo }}\end{array}$ & $\delta$ & $P_{\mathrm{D}}$ & $P_{\text {FA }}$ \\
\hline \multirow{2}{*}{1} & 1.8874 & 0.6133 & 0.2247 \\
\hline & $\overline{2.4379}$ & $\overline{0.4957}$ & $\overline{0.0788}$ \\
\hline \multirow[t]{2}{*}{2} & $\frac{0.7477}{1.7553}$ & $\frac{0.9613}{0.4615}$ & $\frac{0.8986}{0.3634}$ \\
\hline & $\overline{1.7553}$ & $\overline{0.4615}$ & $\overline{0.3634}$ \\
\hline \multirow[t]{2}{*}{3} & 1.5350 & 0.7790 & 0.3919 \\
\hline & 2.2673 & $\begin{array}{l}0.5641 \\
0.7182\end{array}$ & $\begin{array}{l}0.0969 \\
0.3311\end{array}$ \\
\hline 4 & $\overline{2.0085}$ & $\overline{0.6410}$ & 0.1844 \\
\hline \multirow{2}{*}{5} & $\underline{0.7918}$ & 0.9392 & 0.8733 \\
\hline & $\overline{2.1682}$ & $\overline{0.4188}$ & $\overline{0.1777}$ \\
\hline \multirow[t]{2}{*}{6} & 2.7407 & $\underline{0.2210}$ & 0.0591 \\
\hline & $\overline{2.4049}$ & $\overline{0.3761}$ & 0.0888 \\
\hline \multirow[t]{2}{*}{7} & 1.7222 & $\underline{0.6796}$ & 0.2787 \\
\hline & $\overline{1.6011}$ & $\overline{0.8120}$ & $\overline{0.4502}$ \\
\hline \multirow{2}{*}{ NDVI } & 1.5185 & $\underline{0.8122}$ & 0.4645 \\
\hline & 1.7828 & $\overline{0.7778}$ & 0.3180 \\
\hline
\end{tabular}

The best possible results for the band differencing scheme is given in Table II from which it is evident that CUSUM outperforms band differencing. The band differencing approach does not perform well in the Gauteng case study (band 4 performed the best), probably because band differencing needs high spatial correlation to be effective. In the Limpopo case study (NDVI performed the best), the band differencing does not perform comparatively worse when compared to CUSUM, since the Limpopo data set contains a larger amount of spatial correlation.

\section{CONCLUSION}

In this paper, a simple but effective land cover change detection algorithm was presented. Firstly in an off-line optimization phase the CUSUM threshold $h$ that shows the highest $P_{\mathrm{D}}$ and lowest $P_{\mathrm{FA}}$ is determined, for each band. Second, in the operational phase, the time-series CUSUM statistic of band $b$ is computed per pixel and is compared to the threshold $h$ to yield a change or no-change decision. The method was effectively used to determine the location of new settlement developments in the Gauteng and Limpopo provinces of South Africa. The CUSUM algorithm outperformed band differencing in both case studies. For classes containing a definite long term trend, the algorithm could be adapted to construct 45 densities for each year.

\section{REFERENCES}

[1] D. Lu, P. Mausel, and E. Brondizio, E. Moran, "Change detection techniques," International Journal of Remote Sensing, vol. 25, no. 12, pp. 2365-2407, Jun. 2004.

[2] R. Lunetta, J. Knight, J. Ediriwickrema, J. Lyon, and L. Worthy, "Landcover change detection using multi-temporal MODIS NDVI data," Remote Sensing of Environment, vol. 105, no. 2, pp. 142-154, Nov. 2006.

[3] R. Lunetta, J. Ediriwickrema, D. Johnson, J. Lyon, and A. McKerrow, "Impacts of vegetation dynamics on the identification of land-cover change in a biologically complex community in North Carolina, USA," Remote Sensing of Environment, vol. 82, no. 2-2, pp. 258-270, Oct. 2002.

[4] R. Lunetta, R. Alvarez, C. Edmonds, J. Lyon, C. Elvidge, R. Bonifaz, and C. Garca, "NALC/Mexico land-cover mapping results: implications for assessing landscape condition," International Journal of Remote Sensing, vol. 23, no. 16, pp. 3129-3148, Aug. 2002.

[5] W. Kleynhans, B. Salmon, J. Olivier, K. Wessels, and van den Bergh F., "An autocorrelation analysis approach to detecting land cover change using hyper-temporal time-series data," in Proceedings of IEEE Geoscience and Remote Sensing Symposium, Vancouver, Canada, July 2011.

[6] S. Boriah, "Time series change detection: Algorithms for land cover change," Ph.D. dissertation, Graduate School of the University of Minnesota, Apr., 2010.

[7] W. Kleynhans, J. Olivier, K. Wessels, B. Salmon, van den Bergh F., and K. Steenkamp, "Detecting Land Cover Change Using an Extended Kalman Filter on MODIS NDVI Time-Series Data," IEEE Geoscience and Remote Sensing Letters, vol. 8, no. 3, pp. 507-511, Apr. 2011.

[8] B. P. Salmon and et al., "The use of a multilayer perceptron for detecting new human settlements from a time series of MODIS images," International Journal of Applied Earth Observation and Geoinformation, vol. 13, no. 6, pp. 873-883, 2011.

[9] E. Page, "Continuous inspection schemes," Biometrika, vol. 41, pp. 241257, 1954.

[10] J. Kučera, P. Barbosa, and P. Strobl, "Cumulative Sum Charts - A Novel Technique for Processing Daily Time Series of MODIS Data for Burnt Area Mapping in Portugal," in International Workshop on the Analysis of Multi-temporal Remote Sensing Images, Leuven, August 2007.

[11] J. Verbesselt, R. Hyndman, A. Zeileis, and D. Culvenor, "Phenological change detection while accounting for abrupt and gradual trends in satellite image time series," Remote Sensing of Environment, vol. 114, no. 12 , pp. 2970-2980, 2010.

[12] T. L. Grobler, E. R. Ackermann, A. J. van Zyl, J. C. Olivier, and W. Kleynhans, "Land Cover Separability Analysis of MODIS Time Series Data using a combined Simple Harmonic Ossilator and a mean reverting stochastic process," IEEE Journal of Selected Topics in Applied Earth Observations And Remote Sensing, in press, DOI: 10.1109/JSTARS.2012.2183118.

[13] H. Poor and O. Hadjiliadis, Quickest Detection. Cambridge University Press, 2009.

[14] E. R. Ackermann, "Sequential land cover classification," Master's thesis, University of Pretoria, 2011. 